\title{
A Revolution in Tooth Regeneration and Evaluation in Dental, Practice. A Review
}

\section{Gamal Eldeen Zulhemma Elsharkawy}

Professor, Dental Anatomy and Oral Histology Division, Oral Diagnostic Science Dept., Faculty of Dentistry, King Abdul-Aziz University Jeddah, Saudi Arabia and Faculty of Oral and Dental Medicine, Cairo University, Egypt.

$$
\text { Received: } 05 \text { April 2020 / Accepted 10 June 2020 / Publication date: } 25 \text { June } 2020
$$

\begin{abstract}
Recently, therapeutic strategies for tooth regeneration have shifted to cell-based approaches. Embryologically, teeth are ectodermal organs derived from sequential reciprocal interactions between oral epithelial cells and cranial neural crest-derived mesenchymal cells. Teeth are a unique and complex organ and composed of both hard (dentin and enamel) and soft (pulp and periodontium) tissues. Throughout life, healthy teeth have a number of different types of stem cells that play a key role in the regenerative capacity of this tissue. Tooth loss is the most common organ failure. Can a tooth be regenerated? Can adult stem cells be orchestrated to regenerate tooth structures such as the enamel, dentin, cementum and dental pulp, or even an entire tooth? If not, what are the therapeutically viable sources of stem cells for tooth regeneration? Do stem cells necessarily need to be taken out of the body, and manipulated ex vivo before they are transplanted for tooth regeneration? How can regenerated teeth be economically competitive with dental implants? Would it be possible to make regenerated teeth affordable by a large segment of the population worldwide? The typical treatment for irreversibly inflamed/necrotic pulp tissue is root canal treatment. As an alternative approach, regenerative endodontics aims to regenerate dental pulp-like tissues using two possible strategies: cell transplantation and cell homing. The former requires exogenously transplanted stem cells, complex procedures and high costs; the latter employs the host's endogenous cells to achieve tissue repair/regeneration, this review article explores existing and visionary approaches that address some of the above-mentioned questions. Tooth regeneration represents a revolution in dentistry and stomatology as a shift in the paradigm from repair to regeneration: repair is by metal or artificial materials whereas regeneration is by biological restoration. Tooth regeneration is an extension of the concepts in the broad field of regenerative medicine to restore a tissue defect to its original form and function by biological substitutes
\end{abstract}

Keywords: Regeneration stem cell, scaffold, transplantion cell homing

\section{Introduction}

A tooth is a major organ and consists of multiple tissues. The hard tissues of the tooth include the enamel, dentin and cementum. The only vascularized tissue of the tooth is dental pulp that is encased in the mineralized dentin (Poole, 1967). Life ends for a number of wildlife species after loss of complete dentition (Cuozzo and Sauther, 2004). In humans, tooth loss can lead to physical and mental suffering that compromise an individual's self-esteem and quality of life (Pihlstrom et al., 2005). Contemporary dentistry or stomatology restores mis- sing teeth by dentures or dental implants. Whereas dental implants are becoming favorite choices in developed countries, a large segment of the world population, fre- quently in developing countries, cannot afford dental implants. Dental implants, despite being the currently preferred treatment modality, can fail and will not adapt with surrounding bone that necessarily remodels through- out life (Ferreira et al., 2007). A comparison of dental implants and regenerated teeth is provided in Table 1. Dental pro- fession has had the longstanding aspiration to regenerate teeth (Modino et al., 2005; Young et al., 2005; Mao et al., 2006).

Corresponding Author: Gamal Eldeen Zulhemma Elsharkawy, Professor, Dental Anatomy and Oral Histology Division, Oral Diagnostic Science Dept., Faculty of Dentistry, King Abdul-Aziz University Jeddah, Saudi Arabia and Faculty of Oral and Dental Medicine, Cairo University, Egypt. E-mail: Gzhmad@kau.edu.sa 
Table 1: Comparison of current dental treatments including dental implants and dentures with tooth regeneration

\begin{tabular}{lll}
\hline Items of comparison & \multicolumn{1}{c}{ Dental implants } & Tooth regeneration \\
\hline \multirow{2}{*}{ Materials Bone grafting } & Artificial materials & $\begin{array}{l}\text { Regenerated tissues. Stimulates bone } \\
\text { regeneration along with tooth } \\
\text { regeneration. }\end{array}$ \\
& $\begin{array}{l}\text { Metal fails to remodel } \\
\text { with }\end{array}$ & $\begin{array}{l}\text { Regenerated periodontal bone } \\
\text { remodels with existing alveolar bone }\end{array}$ \\
hemodeling potential & $\begin{array}{l}\text { Aseptic loosening or } \\
\text { infections, leading } \\
\text { tofailure }\end{array}$ & $\begin{array}{l}\text { Regenerated teeth have native defense } \\
\text { in dental pulp and perio- } \\
\text { dontal tissue }\end{array}$ \\
\hline
\end{tabular}

\section{Barriers of tooth regeneration towards clinical applications}

For the regeneration of the entire tooth or tooth elements, we are ingrained to believe that stem cells and/or other cells must be transplanted. When tissue engineering was initiated as an interdisciplinary approach to heal tissue defects, three key components were pro- posed: cells, biomaterial scaffolds and signaling factors (Langer and Vacanti, 1993). There is no question that cells, including stem/ progenitor cells, play central roles in tissue regeneration. However, do cells (including stem/progenitor cells) necessarily need to be taken out of the body, manipulated ex vivo and transplanted back into the patient?

Tooth regeneration by cell transplantation is a meritorious approach. However, there are hurdles in the translation of cell-delivery-based tooth regeneration into therapeutics. The most important one of these difficulties is inaccessibility of autologous embryonic tooth germ cells for human applications (Ikeda et al., 2009). Xenogenic embryonic tooth germ cells (from non-human species) may elicit immune rejection and tooth dysmorphogenesis. Autologous postnatal tooth germ cells (e.g. third molars) or autologous dental pulp stem cells are of limited availability and remain uncertain as a cell source to regenerate an entire tooth. Regardless of cell source, cell- delivery approaches for tooth regeneration, similar to cell-based therapies for other tissues, encounter translational barriers. The costs of commercialization process and difficulties in regulatory approval in association with ex vivo cell manipulation have precluded any significant clinical translation effort to date in tooth regeneration (Table 2). As in tissue engineering of other biological structures, regeneration of an entire tooth or various tooth structure, including the enamel, dentin, cementum and dental pulp, by cell transplantation encounters a number of scientific, translational and regulatory difficulties (Mao et al., 2008).

Table 2: Comparison of cell transplantation vs. cell homing approaches for tooth regeneration

\begin{tabular}{lcc}
\hline Items of comparison & $\begin{array}{c}\text { Regeneration by cell } \\
\text { transplantation }\end{array}$ & Regeneration by cell homing \\
\hline Isolation of cell from patient & Yes-autologous cell & No \\
Ex vivo cell manipulation & Yes & No \\
Cell transplantation & Yes & No \\
Develop into off-shell product & Difficult & Possible \\
Cost & High & Not as high \\
\hline
\end{tabular}

\section{Tooth regeneration by cell transplantation}

Table 3 provides a summary of various cell sources that have been used for tooth regeneration. Disassociated cells of porcine or rat tooth buds in biomaterials yielded putative dentin and enamel organ (Young et al., 2002; Duailibi et al., 2004). Tooth bud cells and bone marrow osteoprogenitor cells in collagen, PLGA or silk-protein scaffolds induced putative tooth-like tissues, alveolar bone and periodontal ligament (Young et al., 2005; Duailibi et al., 2008). Embryonic oral epithelium and adult mesenchyme together up-regulate odontogenesis genes upon mutual induction, and yielded dental structures upon transplantation into adult renal capsules or jaw bone (Ohazama et al., 2004). Similarly, implantation of E14.5 rat molar rudiments into adult mouse maxilla produced tooth-like 
structures with surrounding bone (Mantesso and Sharpe, 2009). Multipotent cells of the tooth apical papilla in tricalcium phosphate in minipig incisor extraction sockets generated soft and mineralized tissues of the root (Sonoyama et al., 2006). Dental bud cells from unerupted molar tooth of a 1.5month-old swine were expanded and then seeded in gelatin-chrondroitin-hyaluronan-tri-copolymer scaffold. Cell-seeded scaffolds were implanted autologously in the swine's tooth extraction socket. Thirty-six weeks after implantation, dentin/pulp-like complex structures were identified with odontoblast-like cells and blood vessels in the pulp and appearance of cellular cementum, however the regenerated teeth were much smaller in size than the normal teeth in the same host (Xu et al., 2008). Dental pulp is a vascularized tissue encapsulated in highly mineralized structures including dentin, enamel and cementum, and maintains homeostasis of the tooth as a viable biological organ.

\section{Pulp regeneration by transplantation}

Recent advances in stem cell biology have aided stem cell therapy to regenerate the pulp/dentin complex for conservation and complete structural and functional restoration of the tooth by the triad of tissue engineering: 1) mesenchymal stem cells (MSCs), 2) growth/differentiation factors $r$ cytokines, and migration/homing factors, and 3) the microenvironment (scaffold, extracellular matrix)(Nakashima and Reddi, 2003). We have demonstrated complete pulp regeneration by harnessing autologous dental pulp stem cell (DPSCs) subsets transplanted with stromal cell-derived factor 1 (SDF1) in a collagen scaffold into a canine pulpitis model (Nakashima and Iohara, 2011; Iohara et al., 2011). Next, a novel isolation method was developed employing an optimal granulocyte colony stimulating factor (G-CSF)-induced mobilization of DPSCs for clinical-grade mesenchymal stem cells from a small amount of pulp tissue by good manufacturing practice (GMP)-grade guidelines (Murakami et al., 2013). G-CSF was already approved by the Food and Drug Administration (FDA) for clinical use. The isolated human mobilized DPSCs (MDPSCs) were characterized further by the higher migratory activity and trophic effects including migration, antiapoptosis, and immunosuppression compared with colony-derived DPSCs in vitro. Furthermore, human MDPSCs demonstrated higher regeneration potential using an ectopic tooth root transplantation in severe combined immunodeficient (SCID) mice. Thus, MDPSCs have potential utility for pulp regeneration ((Ikeda et al., 2009). G-CSF was evaluated as an optimal GMP-grade migration/ homing factor for pulp regeneration, having a variety of effects including anti-apoptosis on the transplanted and migrated cells, engraftment of the transplanted cells, angiogenesis, and immunosuppression (Iohara et al., 2013). The potential stem cell therapy for pulpitis harnessing MDPSCs with G-CSF was then examined in a preclinical study. Initially, the human MDPSCs isolated in a totally enclosed system in a GMP-compliant facility were evaluated by their karyotype, safety, and efficacy. Then, canine MDPSCs were isolated by the similar standard operating procedure (SOP) used in humans, and the preclinical feasibility, safety, and efficacy of pulp regeneration was established by autologous transplantation of the MDPSCs with GMP-grade G-CSF into the pulpectomized tooth in a canine pulpitis model (Iohara et al., 2013). On the basis of these preclinical safety and efficacy results and its mechanism for pulp regeneration, the protocol of a clinical study for pulp regenerative therapy was developed and approved by Institutional Review Boards and by the Japanese Ministry of Health, Labor and Welfare.

\section{Periodontal tissue regeneration by transplantation.}

Various approaches have been developed to restore the structure and function of destroyed periodontium, the final goal of periodontal therapy, and treatments, including bone grafting, guided tissue regeneration, and enamel matrix derivatives, have already been approved for clinical use. However, complete regeneration is rarely accomplished by these methods (Esposito et al., 2010). Progress in tissue engineering has offered a new option to supplement existing treatment regimens for periodontal disease. Furthermore, the discovery of progenitor/stem cells residing in the periodontium raises the possibility of restoring damaged periodontal tissues by recruiting their latent regenerative potential. Owing to the difficulties encountered in isolating specialized cells and the associated morbidity involved, stem cells serve as a better alternative.

Research in tissue engineering has shown the therapeutic advantages of delivering stem cells and growth factors in biodegradable scaffolds, which supply the necessary environment to recreate a suitable niche for cellular proliferation and differentiation (Chen, and Jin, 2010).Periodontal ligament 
stem cells (PDLSCs) with characteristics of putative mesenchymal stem cells (MSCs) represent a promising cell-based therapy in reconstructive dentistry for the treatment of periodontal disease (Mrozik et al., 2010). It can be isolated from periodontal ligament (PDL) cells, providing a unique reservoir of stem cells from an accessible tissue resource. Once isolated, PDLSCs can be expanded sufficiently in vitro and the complex differentiation processes involved in periodontal regeneration can be optimized in the right location. The transplantation of ex vivo-expanded PDLSCs was suggested to hold promise as a therapeutic approach for the reconstruction of tissues destroyed by periodontal diseases (Seo et al., 2004). The use of autologous PDLSCs to treat periodontal defects in animal models of periodontitis has further demonstrated the feasibility of using PDLSC-mediated tissue engineering to treat periodontal diseases (Liu et al., 2008). Cells, scaffolds, and growth factors are the three main factors for creating a tissue-engineered construct, and incorporation of DNA into tissue-engineering matrices and its subsequent sustained release may provide an optimal means to engineer tissues (Zhang et al., 2006). The biomaterial based gene transfer method that combines gene therapy and tissue engineering to promote tissue regeneration has been developed. Periodontal tissue engineering using ex vivo gene transfer has been reported to offer a safe new approach for repairing periodontal defects(Chang et al., 2009).

\section{Tooth regeneration by cell homing}

As an initial attempt to regenerate teeth, we first fabricated an anatomically shaped and dimensioned scaffold from biomaterials, using our previously reported approach (Lee et al., 2009; Stosich et al., 2009). The dimensions of the permanent mandibular first molar were derived from textbook averages and therefore IRB exempt. Scaffolds with the shape of the human mandibular first molar ((Figure 2A, B, C, D). Cell homing offers an alternative, especially regarding clinical translation, to previous meritorious methods of tooth regeneration by cell transplantation. The omission of cell isolation and ex vivo cell manipulation accelerates regulatory, commercialization and clinical processes (Lee et al., 2010). The cost of cell-homing - based tooth regeneration is not anticipated to be as robust as cell delivery with regard to both commercialization process and as a treatment cost to the patient. Cell homing is an under-recognized app roach in tissue regeneration (Mao et al., 2010). Here, all cells in growth-factor delivery or growth-factor-free scaffolds are host derived endogenous cells. Tissue genesis requires condensation of sufficient cells of correct lineages (Yelick and Vacanti, 2006).
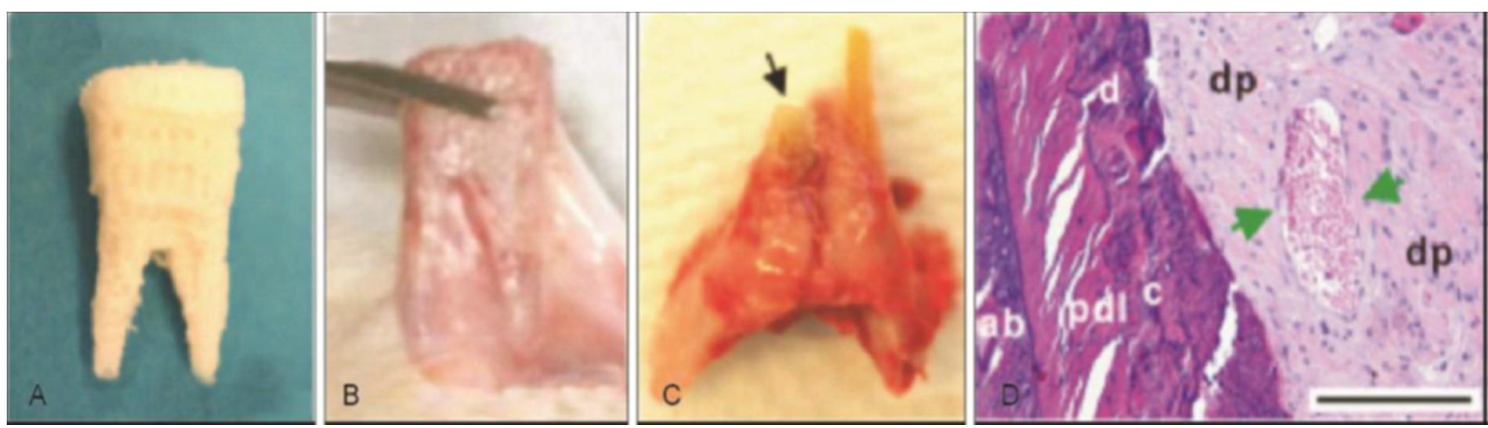

Fig. 2: Tooth regeneration by cell homing. (A) A 3D biomaterials scaffold was fabricated by layer by layer fabrication via bioprinting. In a clinical setting, a patient's missing tooth can be reconstructed by multi-slice imaging using CT or MRI of the contralateral, normal tooth or from anatomic averages. Microchannels are built in the 3D biomaterial human tooth shaped scaffold and serve as conduits for cell recruitment and vascularization. (B) Harvest of human shaped tooth scaffold following 9-week in vivo implantation. (C) A rat shaped tooth scaffold was implanted to replace the rat lower incisor that was freshly extracted. (D) Harvest of regenerated tooth scaffold showed the formation of multiple dental tissues including newly formed alveolar bone (ab), periodontal ligament-like tissue (pdl), dentin-like tissue (d) and dental pulp-like tissue (dp) with blood vessels (arrows). Bar: $200 \mu \mathrm{m}$. 


\section{Dental pulp regeneration via cell homing}

Dental pulp trauma or infections, frequently manifested as pulpitis, are commonly treated by removing the pulp and replacing it with inorganic materials (gutta-percha and sealer cement) via root canal treatment (RCT). Pulp extirpation may make endodontically treated teeth brittle, susceptible to postoperative fractures and reinfections due to coronal leakage or periapical microleakage (Ingle and Bakland, 2002; Dammaschke et al., 2003). Moreover, they lose pulpal sensation to hot/cold stimulation and the ability to detect secondary infections (Caplan et al., 2005). As an alternative approach, regenerative endodontics aims to replace inflamed/necrotic pulp tissue with regenerated pulp-like tissue. Two strategies can be applied towards dental pulp regeneration: cell transplantation and cell homing. The former approach is cell-based, which means transplanting exogenous stem cells loaded onto scaffolds incorporated with signaling molecules into the root canal system of the host to allow regeneration. The transplanted cells are collected from the host (autologous) or from other individuals (allogenic) and may be either processed (separation from tissues) or grown in cultures to increase their numbers. In this case, stem cells are the key for tissue regeneration. About 10 years after the discovery of dental pulp stem cells (Gronthos et al., 2000), pulp/dentine regeneration was accomplished using exogenously transplanted dental stem cells in small and large animals (Huang et al., 2010; Iohara et al., 2011). However, cell-based therapy faces many hurdles in clinical translation because complex procedures need to be followed, such as tooth extraction, pulp extirpation, in vitro cell culture, selection of stem/progenitor cell populations, ex vivo cell expansion,storage and shipping. Also, there are other concerns including potential contamination and development of tumorigenesis during ex vivo cell manipulation (Yildirim et al., 2011; Kim et al., 2012) .Therefore, cell transplantation for pulp regeneration therapy is likely to suffer from a lack of clinical viability, difficulty with regulatory approval and the high costs, besides the risks of immune rejection, pathogen transmission and tumorigenesis during engraftment. Despite its scientific validity, dental pulp regeneration using cell transplantation is unlikely to be economically viable or competitive with current RCT or dental implants. From a therapeutic viewpoint, cell homing may circumvent many of the challenges associated with cell transplantation. In tissue regeneration, cell homing is defined as active recruitment of endogenous cells, including stem/progenitor cells, into an anatomic compartment (Laird et al., 2010; Mao et al., 2010). The concept of cell homing is to achieve tissue repair/regeneration through chemotaxis of host endogenous cells to injured tissue via biological signaling molecules. Compared with stem cell transplantation, cell homing strategies might be easier to perform clinically, because there is no need to isolate and manipulate stem cells in vitro (Kim et al., 2013; Huang and Garcia-Godoy, 2014; Xiao and Nasu, 2014).

\section{Periodontal Ligament Regeneration via cell homing (Scaffolds and Stem Cells).}

The PDL fibers connect the cementum on the tooth root surface to the alveolar bone and fix the tooth in the alveolar socket to attenuate the occlusal stresses. The regeneration of PDL is an important requirement for periodontal regeneration. The ideal outcome would be that the regenerated highlyorganized collagen fibers could re-insert perpendicularly and firmly attached to the regenerated cementum and new bone (Zhu et al., 2015).Inflammation in the periodontal pocket can change the cell biology in the pathological periodontium. Once it is damaged, the periodontium has an only limited capacity for regeneration, which relies on the availability of MSCs. Several types of MSCs remain and are responsible for tissue homeostasis, serving as a source of renewable progenitor cells to generate other required cells throughout adult life. In addition, studies to date have shown that periodontal stem cells can be transplanted into periodontal defects with no adverse immunologic or inflammatory consequences. Therefore, periodontal regeneration relies on the successful recruitment of locally-derived renewable progenitor cells to the lesion site for tissue homeostasis and subsequent diffrentiation into PDL, cementum and bone-forming cells (Bartold et al., 2006). New PDL like tissues were successfully formed via the delivery of stem cells to the defect sites (Mitsiadis et al., 2012; Catón et al., 2011), including the delivery of periodontal ligament stem cells(PDLSCs), bone marrow mesenchymal cells(BMMSCs) (Du et al., 2014), adipose-derived stem cells (ADSCs) (Mohammed et al., 2018), and induced pluripotent stem cells (iPSCs) (Duan et al., 2011). PDLSCs were cultured and osteogenically induced and then seeded on a biphasic calcium phosphate scaffold (BCP), then the PDLSC-seeded scaffolds were transplanted into six dogs. The results showed that the transplantation of PDLSC-seeded BCP promoted effective periodontal regeneration, including new 
bone formation and PDL with reorganized and reborn collage fibers inserting into adjacent cementum and bone at the right angle, along with abundant blood vessels at 12 weeks. Therefore, PDLSC-seeded scaffolds were a promising method for periodontal regeneration. However, several implantations of bone substitute materials into the periodontal wounds produced a long junctional epithelium (LJE) but did not have the ability to regenerate a real periodontium (Bosshardt and Sculean, 2009). As shown in Figure 3, the formation of an LJE only reduced the periodontal pocket depth but had no regeneration of PDL fibers (Figure 3b). In contrast, ideal periodontal tissue regeneration, needed well-organized fibers attaching to the adjacent new cementum and bone (Figure 3c) (Siaili et al., 2018). A barrier membrane was used to maintain the space between the defect and the root surface to enhance the proliferation of PDLSC and the synthesis of both PDL and bone (Figure 3e) (Siaili et al., 2018). Animal studies showed periodontal regeneration histologically (Figure $3 \mathrm{f}-\mathrm{h}$ ) (Bosshardt and Sculean, 2009). Therefore guided tissue regeneration, GTR could guide the soft tissue regeneration without down-growth into the bone defects, thereby promoting the regeneration of the periodontium (Bottino et al., 2012). Non-resorbable materials were prone to be exposed to the oral environment to increase the risk of post-operative infection (Cash, 2019).

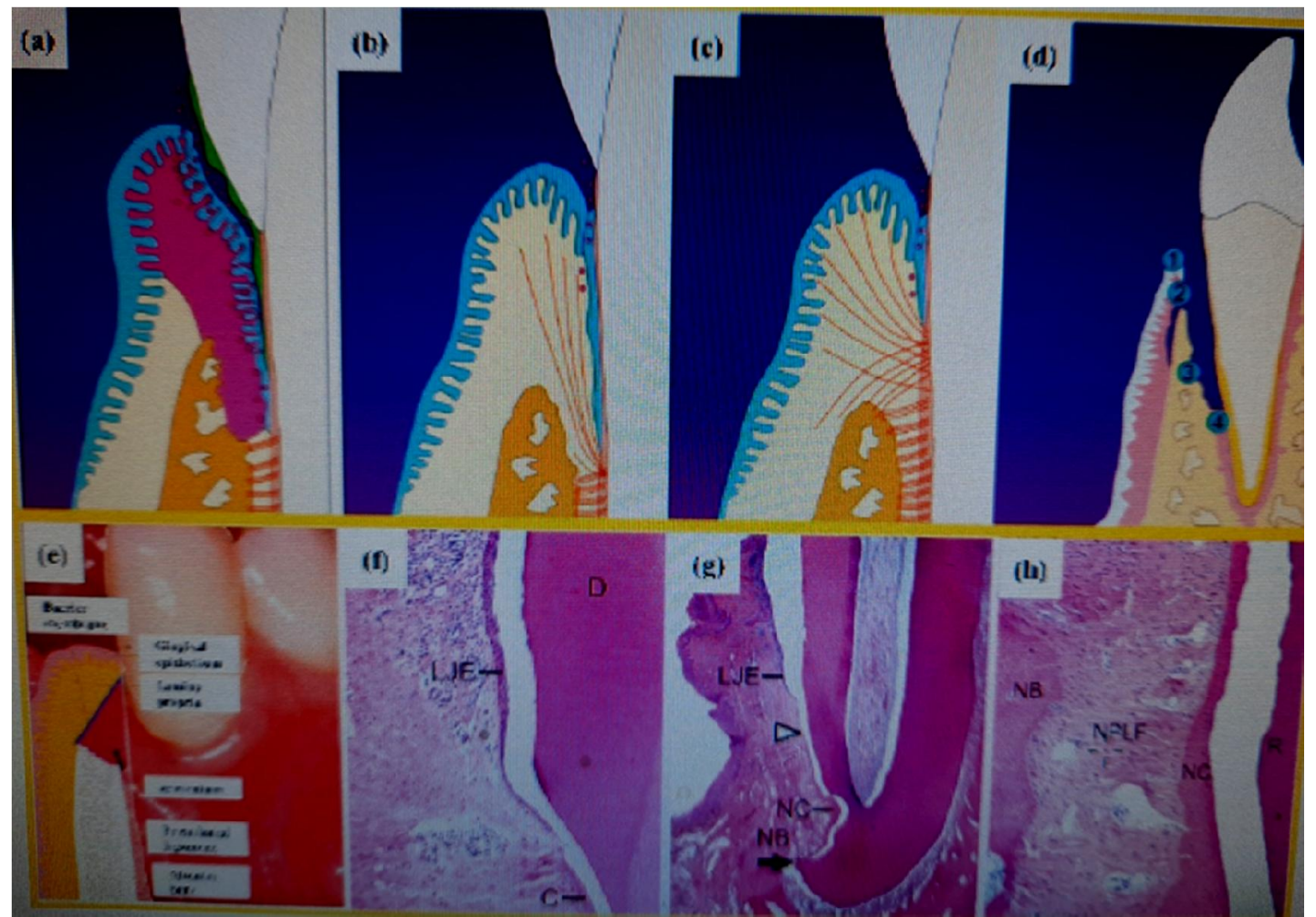

Fig. 3: Periodontal regeneration. (a) inflamed soft tissue and bone resorption in periodontitis; (b) periodontal long junctional epithelium (LJE) repair; (c) ideal periodontal regeneration; (d) schematic of the four compartments from which cells could grow into periodontal wound and repopulate the root surface after periodontal treatment: $\mathrm{O} 1$ oral gingival epithelium; $\mathrm{O} 2$ gingival connective tissue; O3 bone; O4 PDL; (e) schematic of guided tissue regeneration (GTR). (f) optical micrograph shows LJE ending at the coronal-most end of the regenerated cementum (C) and dentin (D); (g) LJE and partial periodontal regeneration, indicated by the formation of new cementum (NC) and new bone (NB). The arrowhead indicates the apical end of the junctional epithelium, whereas the arrow shows the apical border of the defect. (h) optical micrograph showing periodontal regeneration, with the formation of new PDL fibers (NPLF) attaching to both NB and NC. R: root.

In most periodontal regeneration studies, the quality of the attachment function was questionable, because the newly-formed cementum was cellular intrinsic fiber cementum (CIFC), instead of the 
desired AEFC. The numerical density of the inserting fibers in CIFC was low, and the interfacial tissue bonding appeared to be weak (Foster et al., 2012). Several cementum-specific proteins were shown to promote new cementum and bone formation for the damaged periodontal tissues (Arzate et al., 2015). These proteins included cementum-derived growth factor (CDGF), cementum attachment protein (CAP) and cementum protein-1 (CEMP1). They could induce several signaling pathways associated with mitogenesis, increase the concentration of cytosolic $\mathrm{Ca}^{2+}$ activate the protein kinase $\mathrm{C}$ cascade, and promote the migration and preferential adhesion of progenitor cells. These actions could result in the cementoblast and osteoblast differentiation and the production of a mineralized extracellular matrix resembling the cementum (Arzate et al., 2015). The stem cells in the PDL, gingiva, and alveolar bone served as sources for cementoblast progenitors (Bosshardt et al., 1996; producing cementum-specific markers and cementum-like mineralized nodules in culture (Bar-Kana et al., 1998). Indeed, PDLSCs, stem cells from the dental follicle (DFSCs), and adipose derived stem cells(ADSCs) were all able to diffrerentiate into cementoblasts and regenerate the periodontium to form cementum-like tissue, as well as PDL fibers and periodontal vessel regeneration in vivo (Zhu and Liang, 2015).

\section{References}

Arzate, H., M. Zeichner-David and G.Mercado-Celis, 2015. Cementum proteins: Role in cementogenesis,biomineralization, periodontium formation and regeneration. Periodontology 2000, 67, 211-233.

Bar-Kana, I., N. Savion, A. Narayanan and S. Pitaru, 1998. Cementum attachment protein manifestation is restricted to the mineralized tissue forming cells of the periodontium. Eur. J. Oral Sci., 106, 357-364.

Bartold, P.M., S. Shi and S. Gronthos, 2006. Stem cells and periodontal regeneration. Periodontology 2000, 40, 164-172.

Bosshardt, D.D. and A. Sculean, 2009. Does periodontal tissue regeneration really work? Periodontol. 2000, 51, 208-219.

Bosshardt, D.D. and H.E. Schroeder, 1996. Cementogenesis reviewed: A comparison between human premolars and rodent molars. Anat. Rec., 245, 267-292.

Bottino, M.C., V. Thomas, G. Schmidt, Y.K. Vohra, T.M.G. Chu, M.J. Kowolik and G.M. Janowski, Recent advances in the development of gtr/gbr membranes for periodontal regeneration-A materials perspective. Dent. Mater., 28, 703-721.

Caplan, D.J., J. Cai, G. Yin and B.A. White, 2005. Root canal filled versus non-root canal filled teeth: a retrospective comparison of survival times. Journal of Public Health Dentistry, 65, 90-6.

Cash, A., 2019. Years of Guided Bone Regeneration in Implant Dentistry, 2nd ed.; Oxford University Press: Oxford, UK.

Catón, J., N. Bostanci, E. Remboutsika, C. De Bari and T.A. Mitsiadis, 2011. Future dentistry: Cell therapy meets tooth and periodontal repair and regeneration. J. Cell. Mol. Med., 15, 1054-1065.

Chang, P.C., J.A. Cirelli, Q. Jin, Y.J. Seol, J.V. Sugai and N.J. D'Silva, et al., 2009. Adenovirus encoding human platelet-derived growth factor-B delivered to alveolar bone defects exhibits safety and biodistribution profiles favorable for clinical use. Hum Gene Ther., 20:486-96.

Chen, F.M. and Y. Jin, 2010. Periodontal tissue engineering and regeneration: current approaches and expanding opportunities. Tissue Eng Part B Rev. 2010;16:219-55.

Cuozzo, F.P. and M.L. Sauther, 2004.. Tooth loss, survival, and resource use in wild ring-tailed lemurs (Lemur catta): implications for inferring conspecific care in fossil hominids. J Hum Evol., 46: 623-631.

Dammaschke, T., D. Steven, M. Kaup and K.H. Ott, 2003. Longterm survival of root-canal-treated teeth: a retrospective study over 10 years. Journal of Endodontics, 29, 638-43.

Du, J., Z. Shan, P. Ma, S. Wang, Z. Fan, 2014. Allogeneic bone marrow mesenchymal stem cell transplantation for periodontal regeneration. J. Dent. Res., 93, 183-188. 55.

Duailibi, M.T., S.E. Duailibi and C.S. Young, et al., 2004. Bioengineered teeth from cultured rat tooth bud cells. J Dent Res., 83: 523-528.

Duailibi, S.E., M.T. Duailibi and W. Zhang, et al., 2008. Bioengineered dental tissues grown in the rat jaw. J Dent Res., 87: 745-750. 
Duan, X., Q. Tu, J. Zhang, J. Ye, C. Sommer, G. Mostoslavsky, D. Kaplan, P. Yang and J. Chen, 2011. Application of induced pluripotent stem (ips) cells in periodontal tissue regeneration. J. Cell. Physiol., 226, 150-157.

Esposito, M., M.G Grusovin, N. Papanikolaou, P. Coulthard, H.V. Worthington, 2010. Enamel matrix derivative (Emdogain $\AA)$ for periodontal tissue regeneration in intrabony defects. Aust Dent J., 55:101-4.

Ferreira, C.F., R.S. Magini and P.T. Sharpe, 2007. Biological tooth replacement and repair. J Oral Rehabil., 34: 933-939.

Foster, B.L., K.J. Nagatomo, F.H. Nociti Jr., H. Fong, D. Dunn, A.B. Tran, W. Wang, S. Narisawa, J.L. Millán and M.J. Somerman, 2012. Central role of pyrophosphate in acellular cementum formation. PLoS ONE, 7, e38393.

Gronthos, S., M. Mankani, J. Brahim, P.G Robey and S. Shi, 2000. Postnatal human dental pulp stem cells (DPSCs) in vitro and in vivo. Proceedings of the National Academy of Sciences of the United States of America, 97, 13625-30.

Huang, G.T. and F. Garcia-Godoy, 2014. Missing concepts in de novo pulp regeneration. Journal of Dental Research, 93,717-24.

Huang, G.T., T. Yamaza and L.D. Shea et al., 2010. Stem/progenitor cell-mediated de novo regeneration of dental pulp with newly deposited continuous layer of dentin in an in vivo model. Tissue Engineering Part A, 16, 605-15.

Ikeda, E., R. Morita and K. Nakao, et al., 2009. Fully functional bioengineered tooth replacement as an organ replacement therapy. Proc Natl Acad Sci., 106: 13475-13480.

Ingle, J.I. and L.K. Bakland, 2002. Structure and function of the dentin-pulp complex. In: Ingle JI, Bakland LK, eds.Endodontics, $5^{\text {th }}$ edn. Hamilton-London: BC Decker Inc, pp 121-43.

Iohara, K., K. Imabayashi and R. Ishizaka et al., 2011. Complete pulp regeneration after pulpectomy by transplantation of CD105 + stem cells with stromal cell-derived factor-1. Tissue Engineering Part A, 17, 1911-20.

Iohara, K., K. Imabayashi, R. Ishizaka, A. Watanabe, J. Nabekura and M. Ito, et al., 2011. Complete pulp regeneration after pulpectomy by transplantation of CD105+ stem cells with stromal cellderived factor-1. Tissue Eng Part A., 17(15-16):1911-20.

Iohara, K., M. Murakami, N. Takeuchi, Y. Osako, M. Ito, R. Ishizaka, et al., 2013. A novel combinatorial therapy with pulp stem cells and granulocyte colony-stimulating factor for total pulp regeneration. Stem Cells Transl Med., 2:521-33.

Kim, S.G., J. Zhou and L. Ye et al., 2012. Regenerative endodontics: barriers and strategies for clinical translation. Dental Clinics of North America, 56, 639-49.

Kim, S.G., Y. Zheng and J. Zhou et al., 2013. Dentin and dental pulp regeneration by the patient's endogenous cells. Endodontic Topics, 28, 106-17.

Laird, D.J., U.H. von Andrian A.J. Wagers, 2008. Stem cell trafficking in tissue development, growth, and disease. Cell, 132, 612-30.

Langer, R. and J.P. Vacanti, 1993. Tissue engineering. Science, 260: 920-926.

Lee, C.H., J.L. Cook and A. Mendelson, et al., 2010. Regeneration of the articular surface of the rabbit synovial joint by cell homing: a proof of concept study. Lancet, 376: 440-448.

Lee, C.H., N.W. Marion, S. Hollister and J.J. Mao, 2009. Tissue formation and vascularization in anatomically shaped human joint condyle ectopically in vivo. Tissue Eng Part A, 15: 3923-3930.

Liu, Y., Y. Zheng, G. Ding, D. Fang, C. Zhang and P.M. Bartold, et al., 2008. Periodontal ligament stem cell-mediated treatment for periodontitis in miniature swine. Stem Cells, 26:1065-73.

Mantesso, A. and P. Sharpe, 2009. Dental stem cells for tooth regeneration and repair. Expert Opin Biol Ther., 9: 1143-1154.

Mao, J.J., G. Vunjak-Novakovic, A.G. Mikos and A. Atala, 2008. Trans- lational approaches in tissue engineering and regenerative medicine. Boston: Artech House.

Mao, J.J., M.S. Stosich and E.K. Moioli et al., 2010. Facial reconstruction by biosurgery: cell transplantation versus cell homing. Tissue Engineering Part B: Reviews, 16, 257-62.

Mao, J.J., M.S. Stosich and E.K. Moioli, et al. Facial reconstruction by biosurgery: cell transplantation versus cell homing. Tissue Eng Part B Rev., 16: 257-262.

Mao, J.J., W.V. Giannobile and J.A. Helms, et al., 2006. Craniofacial tissue engineering by stem cells. J Dent Res., 85: 966-979. 
Mitsiadis, T.A., W.A. oloszyk and L. Jiménez-Rojo, 2012. Nanodentistry: Combining nanostructured materials and stem cells for dental tissue regeneration. Nanomedicine, 7, 1743-1753.

Modino, S.A. and PT. Sharpe, 2005. Tissue engineering of teeth using adult stem cells. Arch Oral Biol., 50: 255-258.

Mohammed, E.; E.Khalil and D.Sabry, 2018. E_ect of adipose-derived stem cells and their exo as adjunctive therapy to nonsurgical periodontal treatment: A histologic and histomorphometric study in rats. Biomolecules, $8,167$.

Mrozik, K., S. Gronthos, S. Shi and P.M. Bartold, 2010. A method to isolate, purify, and characterize human periodontal ligament stem cells. In: Oral biology. New York: Springer, 269-84.

Murakami, M., H. Horibe, K. Iohara, Y. Hayashi, Y. Osako, Y. Takei, K. Nakata, N. Motoyama, K. Kurita and M. Nakashima, 2013. The use of granulocyte-colony stimulating factor induced mobilization for isolation of dental pulp stem cells with high regenerative potential. Biomaterials. 2013;34:9036-47.

Nakashima, M. and A.H. Reddi, 2003. The application of bone morphogenetic proteins to dental tissue engineering. Nature Biotech., 21:1025-32.

Nakashima, M. and K. Iohara, 2011. Regeneration of dental pulp by stem cells. Adv Dent Res. 2011;23:313-19.

Ohazama, A., S.A. Modino, I. Miletich and P.T. Sharpe, Stem- cell-based tissue engineering of murine teeth. J Dent Res 2004; 83: 518-522.

Pihlstrom, B.L., B.S. Michalowicz and N.W. Johnson, 2005. Periodontal diseases. Lancet, 366: $1809-1820$.

Poole, D. 1967. Structural and chemical organization of teeth. New York: Academic Press,

Seo, B.M., M. Miura, S. Gronthos, P. Mark Bartold, S. Batouli, J. Brahim, et al., 2004. Investigation of multipotent postnatal stem cells from human periodontal ligament. Lancet, 364:149-55.

Shi, H., W. Zong, X. Xu and J. Chen, 2018. Improved biphasic calcium phosphate combined with periodontal ligament stem cells may serve as a promising method for periodontal regeneration. Am. J. Transl. Res., 10, 4030.

Siaili, M., D. Chatzopoulou and D.G. Gillam, 2018. An overview of periodontal regenerative procedures for the general dental practitioner. Saudi Dent. J., 30, 26-37.

Sonoyama, W., Y. Liu and D. Fang, et al., 2006. Mesenchymal stem cell-mediated functional tooth regeneration in swine. PLoS One, 2006; 1: e79.

Stosich, M.S., E.K. Moioli and J.K. Wu, et al., 2009. Bioengineering strategies to generate vascularized soft tissue grafts with sustained shape. Methods, 47: 116-121.

Xiao, L. and M. Nasu, 2014. From regenerative dentistry to regenerativemedicine: progress, challenges, and potential applications of oral stem cells. Stem Cells and Cloning: Advances and Applications 7, 89-99.

Xu, W.P., W. Zhang and R. Asrican, et al., 2008. Accurately shaped tooth bud cell-derived mineralized tissue formation on silk scaffolds. Tissue Eng Part A., 14: 549-557.

Yelick, P.C. and J.P. Vacanti, 2006. Bioengineered teeth from tooth bud cells. Dent Clin North Am., 50: 191-203.

Yildirim, S., S.Y. Fu and K. Kim et al., 2011. Tooth regeneration: a revolution in tomatology and evolution in regenerative medicine. International Journal of Oral Science, 3, 107-16.

Young, C.S. and S.W. Kim and C. Qin, et al., 2005. Developmental analysis and computer modelling of bioengineered teeth. Arch Oral Biol., 50: 259-265.

Young, C.S., H. Abukawa and R. Asrican, et al. 2005. Tissue-engineered hybrid tooth and bone. Tissue Eng., 2005; 11: 1599-1610.

Young, C.S., S. Terada and J.P. Vacanti, et al., 2002. Tissue engineering of complex tooth structures on biodegradable polymer scaffolds. J Dent Res., 81: 695-700.

Zhang, Y., X. Cheng, J. Wang, Y. Wang, B. Shi, C. Huang, et al., 2006. Novel chitosan/collagen scaffold containing transforming growth factor- $\beta 1 \mathrm{DNA}$ for periodontal tissue engineering. Biochem Biophys Res Commun., 344:362-9.

Zhu, W. and M. Liang, 2015. Periodontal ligament stem cells: Current status, concerns, and future prospects. Stem Cells Int., 2015, 972313.

Zhu, W., Q. Zhang, Y. Zhang, L. Cen, and J. Wang, 2015. Pdl regeneration via cell homing in delayed replantation of avulsed teeth. J. Transl. Med., 13, 357. 